\title{
Miniaturization of a microstrip antenna using a compact and thin magneto-dielectric substrate
}

\author{
Farhad Farzami, Student Member, Keyvan forooraghi, Member, and Majid Norooziarab, Student \\ Member, IEEE
}

\begin{abstract}
Miniaturization of a rectangular microstrip antenna using a magneto-dielectric substrate is discussed theoretically and experimentally. A compact magneto- dielectric substrate is designed using a metamaterial structure which can reduce the antenna dimensions by increasing the constitutive parameters of the substrate. Furthermore, the proposed structure is thin enough to be embedded in a single dielectric substrate. The area of the microstrip antenna with the proposed magneto-dielectric substrate at $2.4 \mathrm{GHz}$ is reduced up to about $65 \%$ compared to a conventional dielectric microstrip antenna. The bandwidth of the miniaturized antenna is almost unchanged due to the increase of the magnetic permeability at the designed 2.4 GHz frequency. Finally a fabricated version of the miniaturized antenna is tested and measured. The results of the measurement and simulation are in good agreement.
\end{abstract}

Index Terms-, Magneto-dielectric substrate, Miniaturization, Microstrip patch Antenna.

\section{INTRODUCTION}

$\mathbf{M}^{\prime}$ INIATURIZATION of microstrip antennas has been considered so far in different ways, the most common of which is to use a substrate with a high electric permittivity [1]. In this method, surface waves are excited which reduces the radiation efficiency, degrades antenna radiation pattern and makes difficulties in impedance matching due to the increase in impedance of the patch's edge. Moreover miniaturization of microstrip antennas causes the quality factor (Q) to increase and consequently decreases the bandwidth and the antenna radiation efficiency. Using magneto-dielectric substrates is an alternative method for miniaturization of microstrip antennas. The meta-substrate structure discussed in [2] or [3] employs a relatively large number of parallel dielectric slabs loaded by Split Ring Resonators (SRRs) or spiral loops as the overall substrate. Thus, the substrate structure is obviously hard for

Manuscript received September 13, 2011; revised December 14, 2011; accepted December 19, 2011. The authors are with the Department of Electrical and Computer Engineering, Tarbiat Modares University, Tehran 14115, Iran (email: Farzami.farhad@gmail.com).

Color versions of one or more of the figures in this letter are available online at http://ieeexplore.ieee.org.

Digital Object Identifier implementation and its thickness is limited by the SRRs.

In this paper a magneto-dielectric substrate which can be embedded in the host dielectric substrate is realized. The structure can influence the constitutive parameters of the host dielectric substrate. In this way the magnetic permeability can be increased to compensate for bandwidth reduction while miniaturizing the antenna. Furthermore, the proposed substrate structure is compatible with planar circuitry. Using the magneto-dielectric substrate, a miniaturized microstrip rectangular patch antenna is realized. The microstrip antenna is then fabricated, tested and measured. The measurement results agree well with those obtained from simulations.

\section{MicRostrip ANTENNAS AND THE MAGNETO-DIELECTRIC} SUBSTRATE

The microstrip antenna behaves like a $\lambda / 2$ microstrip transmission line in its dominant mode and radiates from its two edges with two equivalent slots along the resonating dimension, [4]. The length of the microstrip rectangular patch antenna determines the resonance frequency of the antenna. The exact value of resonance depends on factors such as ground dimensions, width of the patch and metal thickness. The approximate dominant resonance frequency can be obtained as

$$
\begin{aligned}
& f=\frac{c}{2(L+h) \sqrt{\varepsilon_{e} \mu_{e}}} \\
& \varepsilon_{e}=\frac{\varepsilon_{r}+1}{2}+\frac{\varepsilon_{r}-1}{2}\left(1+\frac{12 h}{W}\right)^{-0.5}
\end{aligned}
$$

Where $W$ and $L$ are respectively the width and length of the rectangular patch, $\varepsilon_{\mathrm{e}}$ is the effective electric permittivity of a dielectric substrate with a relative permittivity of $\varepsilon_{\mathrm{r}}$ and a thickness of $h$ and $\mu_{\mathrm{e}}$ is effective permeability of the substrate which is equal to $\mu_{0}$ in a dielectric substrate. According to Eq. (1), the resonance frequency of the antenna is reversely proportional to the effective constitutive parameters $\left(\varepsilon_{\mathrm{e}}, \mu_{\mathrm{e}}\right)$. Thus, with the increase of the constitutive parameters, the resonance frequency can be decreased for a fixed dimension which results in antenna miniaturization at a fixed frequency. Metamaterial structures such as SRRs or spiral rings etched 
on host dielectric slabs were employed underneath the patch $[2,3]$. These slabs were aligned so that the magnetic fields were

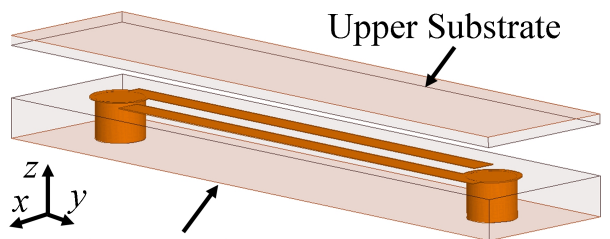

Lower Substrate

Top view
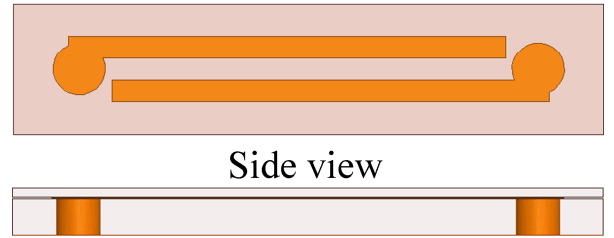

(a)

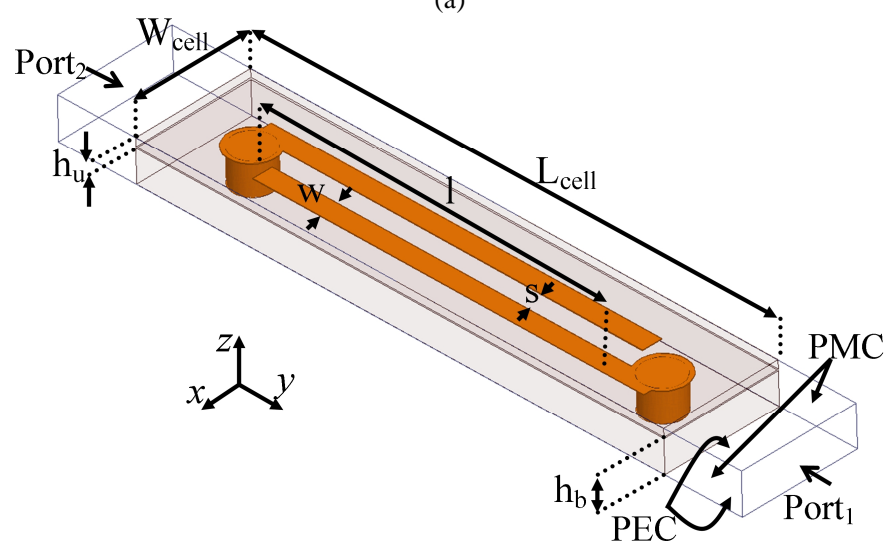

(b)

Fig. 1. Geometry of the proposed unit cell, (a) Top and side view, (b) The specific boundary conditions to extract constitutive parameters. The dimensions are $\mathrm{W}_{\text {cell }}=3, \mathrm{~L}_{\text {cell }}=13.5, \mathrm{l}=10, \mathrm{w}=0.5, \mathrm{~s}=0.5, \mathrm{~h}_{\mathrm{u}}=0.203$ and $\mathrm{h}_{\mathrm{b}}=0.813$, all in $\mathrm{mm}$.

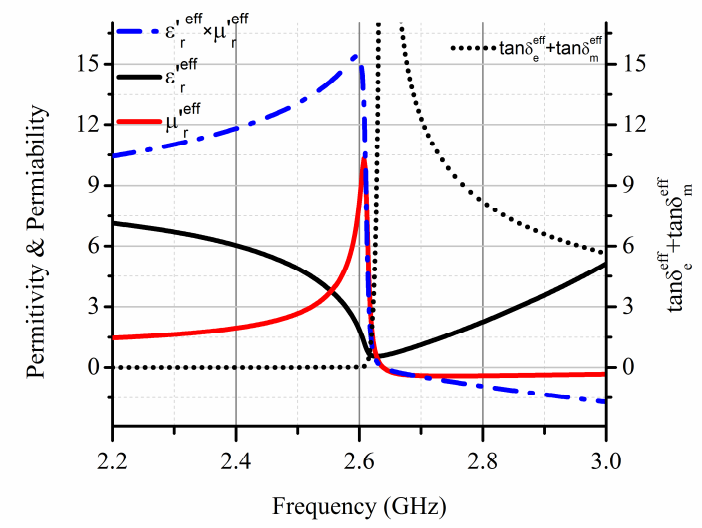

Fig. 2. Effective constitutive parameters of the magneto-dielectric substrate (solid lines) with the electric and magnetic losses (dotted line). The product of the dielectric permittivity and magnetic permeability constants is also shown (dashdot line).

perpendicular to the surface of the rings which induce currents in the rings. The induced currents generate magnetic dipole moments and can change the magnetic permeability of the medium [5].

In this paper a magneto-dielectric substrate which can be used in microstrip antennas is introduced. A unit cell of the proposed substrate is shown in Fig. 1. As can be seen, the

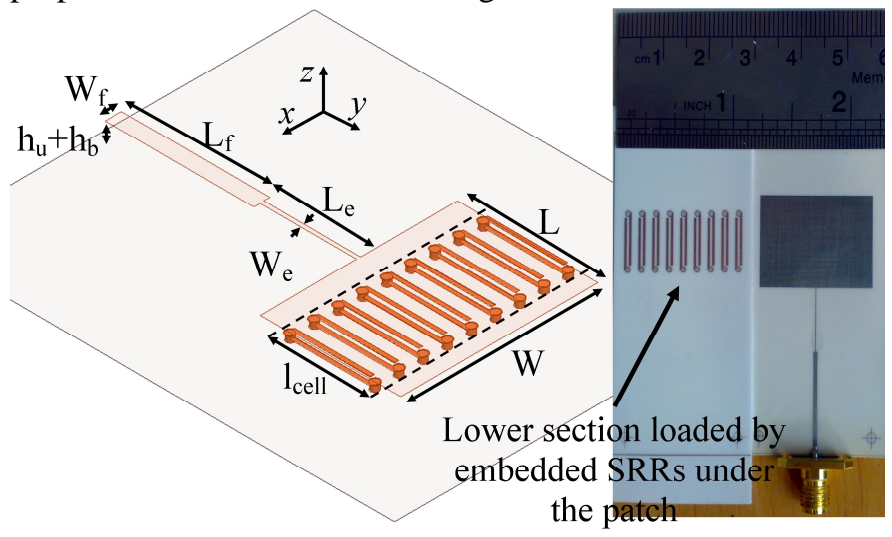

Fig. 3. The microstrip patch antenna with the proposed magneto-dielectric substrate. The fabricated antenna is also shown in the right.

lower substrate is loaded by metallic vias and strips which make a split loop normal to the $\mathrm{x}$ direction forming an SRRlike structure.

The unit cell then contributes to the magneto-dielectric substrate. A row of 9 SRRs is embedded in the lower section and the patch of the antenna is etched on the upper section. In this case the host dielectric is Rogers RO4003 with $\varepsilon_{r}=3.55$ and $\tan \delta=0.0027$. The thickness of the lower section is 0.813 $\mathrm{mm}$ and that of the upper section is $0.203 \mathrm{~mm}$.

Using the method introduced by Smith et al. [6], the constitutive parameters can be calculated from the frequency response of the unit cell under specific boundary conditions, Fig. 1. In this case, the side walls ( $y z$ plane) are perfect magnetic conductor (PMC) and the top and bottom walls ( $x y$ plane) are perfect electric conductor (PEC). The effective constitutive parameters are then extracted which can be attributed to the whole magneto- dielectric substrate, (Fig. 2). The frequency response of metamaterials mostly depends on their dimensions. For the embedded resonators of Fig. 1, the length, $l$, has the most important impact and the resonance frequency of the embedded resonators decreases by increasing the length.

\section{Potentials AND Limitations of THE MAGNETO- DIELECTRIC SUBSTRATE}

In this section a microstrip antenna with the proposed magneto-dielectric substrate is compared with other equivalent substrates. All substrates have the same thickness $\left(h_{u}+h_{b}\right)$ and the resonant frequency of the antennas is fixed at $2.4 \mathrm{GHz}$. The substrates are listed as follows:

1) The proposed magneto-dielectric substrate, (Fig. 3).

2) An equivalent magneto-dielectric substrate with the constitutive parameters obtained by the method of [6]. In this case the substrate is not loaded by the embedded SRRs and it is a homogenous magneto-dielectric substrate modeled by $\varepsilon_{r}^{\text {equ }}(f) \quad=\varepsilon_{r}{ }^{e f f}(f), \quad \mu_{r}{ }^{e q u}(f)=\mu_{r}{ }^{e f f}(f)$, 
$\tan \delta_{e}(f)=\tan \delta_{e} e^{e f f}(f)$ and $\tan \delta_{m}(f)=\tan \delta_{m}{ }^{e f f}(f)$, Fig. 2 (solid curves).

3) A hypothetical dispersive dielectric substrate with $\varepsilon_{r}^{r e f}(f)=$

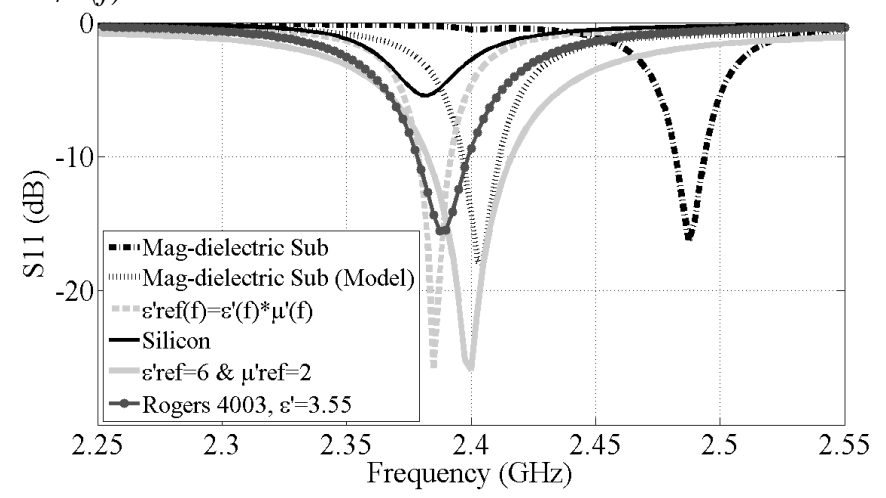

Fig. 4. Return losses of the six cases substrates.

$\varepsilon_{r}^{e f f}(f) \times \mu_{r}^{e f f}(f), \mu_{r}^{r e f}(f)=1$ and $\tan \delta_{e}=0.02$, Fig. 2 (dasheddotted curve).

4) A nondispersive dielectric substrate with $\varepsilon_{r}^{r e f}=\left.\varepsilon_{r}^{e f f}\right|_{f=2.4 \mathrm{GHz}} \times\left.\mu_{r}^{e f f}\right|_{f=2.4 \mathrm{GHz}}=12, \mu_{\mathrm{r}}=1$. In this

case a lossless high permittivity dielectric such as silicon with $\varepsilon_{r}=11.9$ and $\tan \delta_{e}=0$ can be considered.

5) A hypothetical nondispersive magneto-dielectric substrate with

$\varepsilon_{r}^{r e f}=\left.\varepsilon_{r}^{e f f}\right|_{f=2.4 \mathrm{GHz}}=6, \tan \delta_{e}=\left.\tan \delta_{e}^{e f f}\right|_{f=2.4}=0.01$

$\mu_{r}^{r e f}=\left.\mu_{r}^{e f f}\right|_{f=2.4}=2, \tan \delta_{m}=\left.\tan \delta_{m}^{e f f}\right|_{f=2.4}=0.01$ in the

whole frequency range.

6) Rogers RO4003 as the dielectric substrate with $\varepsilon_{r}=3.55$ and $\tan \delta_{e}=0.0027$.

For the first five cases above, the dimensions are $W_{f}=0.8$, $L_{f}=18.5, W_{e}=0.05, L_{e}=12, W=24.5, L=17.5$, all in $\mathrm{mm}$. The dimensions in the last case are $W_{f}=2.3, L_{f}=31.2, W_{e}=0.65$, $L_{e}=19.5, \quad W=41.5, L=32$, all in $\mathrm{mm}$, Fig. 3. Table I summarizes different parameters of the antennas with the above substrates. The return loss of the corresponding antennas is depicted in Fig. 4. All simulations are performed with the full wave HFSS software.

Comparing the first two cases from Table I, it can be inferred that the unit cell of the magneto-dielectric substrate under the specified boundary conditions (case 1) can be well used to extract the constitutive parameters of the magnetodielectric substrate, Fig. 4. The corresponding frequency responses of the two cases are only $82 \mathrm{MHz}$ apart from each other (a 4\% frequency shift). Obviously the radiation efficiency is greater in the second case as the substrate is homogeneous and single section. On the other hand, the air gap between the two sections of the first substrate reduces the gain and shifts the resonance frequency.

The high permittivity lossy dielectric and the high permittivity lossless nondispersive dielectric substrates are investigated in cases 3 and 4, respectively. From Table I, case 3 has lower gain and efficiency compared to the proposed magneto-dielectric substrate (case 1). In case 4 the radiation efficiency is $100 \%$ because the dielectric is lossless, though it suffers from a narrow bandwidth due to the increased quality factor. In fact, the radiation efficiency of the proposed

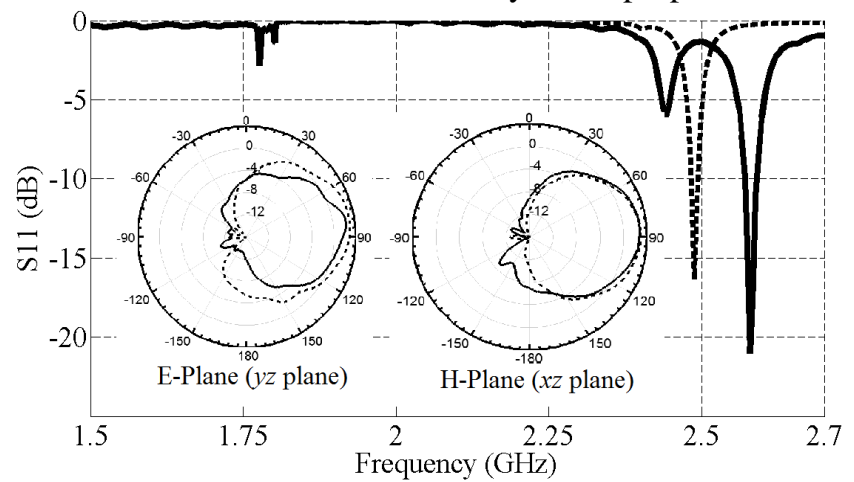

Fig. 5. Return losses and patterns of the simulated (dashed lines) and measured (solid lines) antennas.

magneto-dielectric is more than case 3 and the impedance bandwidth is improved compared to case 4 .

To compare the dispersive and nondispersive magneto dielectric, a hypothetical magneto-dielectric substrate with $\varepsilon_{r}=6-0.01 j$ and $\mu_{r}=2-0.01 j$ is considered in case 5. From table I, it can be concluded that the dispersion free magnetodielectric substrate has much more impedance bandwidth compared to the dispersive magneto-dielectric substrate (case 1). The dispersion effect on magneto-dielectric substrates is extensively studied in [7].

In case 6 , a microstrip antenna with the conventional dielectric substrate Rogers RO4003 is designed. The patch of the microstrip antenna with this substrate is $S_{\text {patch }}^{\text {Rogers }}=41.5 \times 32=1328 \mathrm{~mm}^{2}$, while with the proposed magneto-dielectric substrate is $S_{\text {patch }}^{\text {Mag-die }}=24.5 \times 17.5=429 \mathrm{~mm}^{2}$. A prototype of the fabricated antenna with the magneto-dielectric substrate is shown in the caption of Fig. 3. As can be seen a reduction of about $65 \%$ is achieved while the bandwidth is kept constant at $18 \mathrm{MHz}$, Fig. 5. The E- and H-plane patterns are depicted in Fig. 5, from which it can be seen that the maximum Gain is about $2.8 \mathrm{~dB}$. It should be emphasized that the first resonant frequency of the fabricated antenna is non-radiating and it may be occurred due to practical implementations.

Table I. Results of the six cases of substrates

\begin{tabular}{cccccc}
\hline case & $\mathrm{f}_{0}$ & $\left.\mathrm{BW}\right|_{-6 \mathrm{~dB}}$ & $\left.\mathrm{BW}\right|_{-10 \mathrm{~dB}}$ & $\left.\mathrm{Gain}\right|_{\mathrm{f} 0}$ & $\eta_{\mathrm{r}}=\mathrm{G} / \mathrm{D}$ \\
1 & $2487.7 \mathrm{MHz}$ & $20 \mathrm{MHz}$ & $12 \mathrm{MHz}$ & $3.2 \mathrm{~dB}$ & $45 \%$ \\
2 & $2405.5 \mathrm{MHz}$ & $26 \mathrm{MHz}$ & $14 \mathrm{MHz}$ & $5 \mathrm{~dB}$ & $70 \%$ \\
3 & $2385.5 \mathrm{MHz}$ & $23 \mathrm{MHz}$ & $13 \mathrm{MHz}$ & $2 \mathrm{~dB}$ & $20 \%$
\end{tabular}




\begin{tabular}{cccccc}
\hline 4 & $2375.5 \mathrm{MHz}$ & $8 \mathrm{MHz}$ & - & $7 \mathrm{~dB}$ & $100 \%$ \\
5 & $2375.5 \mathrm{MHz}$ & $83 \mathrm{MHz}$ & $47 \mathrm{MHz}$ & $2 \mathrm{~dB}$ & $34 \%$ \\
6 & $2385.5 \mathrm{MHz}$ & $40 \mathrm{MHz}$ & $20 \mathrm{MHz}$ & $6.25 \mathrm{~dB}$ & $82 \%$
\end{tabular}

Finally, it should be noted that further miniaturization can be achieved in other center frequencies, but at the cost of increased losses. For example, $\varepsilon_{r}^{\prime}$ eff $(f) \times \mu_{r}^{\prime}$ eff $(f)$ has a greater value at $f=2.55 \mathrm{GHz}$ compared to $f=2.4 \mathrm{GHz}$, Fig. 2 ; however the radiation efficiency is reduced due to the increasing electric and magnetic losses. Concerning the effect of the unit cell dimension on the resonance frequency of the embedded resonators, miniaturization can be achieved in any arbitrary center frequency without introducing further losses to the antenna. According to the procedure of designing the antenna in this paper, the proposed magneto-dielectric substrate should have a maximum product of constitutive parameters with minimum losses at the desired center frequency. Now if miniaturization is desired in other frequencies, the frequency response of the embedded resonators must be taken into account for a proper design.

\section{CONCLUSION}

Design of a magneto-dielectric substrate which is highly preferred for planar structures is discussed in this paper. The proposed structure can be used to miniaturize microstrip antennas. Using the proposed magneto-dielectric substrate, a rectangular microstrip antenna is designed at the center frequency of $2.4 \mathrm{GHz}$. The antenna is about one third of a rectangular microstrip antenna with a conventional dielectric substrate while the impedance bandwidth is still remained and it has acceptable radiation efficiency and gain. The constitutive parameters of magneto-dielectric substrate are extracted and the effects of loss and dispersion on radiation and bandwidth characteristics are investigated. Finally, a prototype of the antenna is fabricated and measured. The results of the measurement and simulation are in good agreement.

\section{REFERENCES}

[1] J. S. Colburn and Y. Rahmat-Samii, "Patch antennas on externally perforated high dielectric constant substrates," IEEE Trans. Antennas Propag ,.vol. 47, no. 12, pp. 1785-1794,1999.

[2] P. Mookiah, and K.R. Dandekar, "Metamaterial-Substrate Antenna Array for MIMO Communication System," IEEE Trans. AntennasPropag, VOL. 57, NO. 10, October 2009

[3] H. Mosallaei, and K. Sarabandi, "Design and Modeling of Patch Antenna Printed on Magneto-Dielectric Embedded-Circuit Metasubstrate", IEEE Trans. AntennasPropag, VOL. 55, NO. 1, January 2007.

[4] C. A. Balanis and E. Corporation, Modern antenna handbook: Wiley Online Library, 2008.

[5] C. Caloz, T. Itoh, "Electromagnetic metamaterial: Transmission line theory and microwave application," Hoboken, New Jersey and Canada: Wiley, 2006.

[6] D. R. Smith, D. C. Vier, Th. Koschny and C. M. Soukoulis, "Electromagnetic parameter retrieval from inhomogeneous metamaterials," Physical review E 71, 036617 s2005d
[7] P. M. T. Ikonen, K. N. Rozanov, A. V. Osipov, P. Alitalo, and S. A. Tretyakov, "Magnetodielectric substrates in antenna miniaturization: Potential and limitations," Antennas and Propagation, IEEE Transactions on, vol. 54, pp. 3391-3399, 2006. 